EPJ Web of Conferences 32, 02003 (2012)

DOI: $10.1051 /$ epjconf/20123202003

(C) Owned by the authors, published by EDP Sciences, 2012

\title{
The Real-Time system for MHD activity control in the FTU tokamak
}

C. Sozzi ${ }^{1}$, E. Alessi ${ }^{1}$, L. Boncagni ${ }^{2}$, C. Galperti ${ }^{1}$, C. Marchetto ${ }^{1}$, S. Nowak ${ }^{1}$, W. Bin ${ }^{1}$, A. Botrugno ${ }^{2}$, A. Bruschi ${ }^{1}$, S. Cirant ${ }^{1}$, G. D’Antona ${ }^{3}$, O. D’Arcangelo ${ }^{1}$, M. Davoudi ${ }^{3}$, D. Farina ${ }^{1}$, R. Ferrero ${ }^{1}$, L. Figini $^{1}$, S. Garavaglia ${ }^{1}$, G. Granucci ${ }^{1}$, A. Grosso ${ }^{2}$, F. Iannone ${ }^{2}$, E. Lazzaro ${ }^{1}$, A. Moro ${ }^{1}$, A. Nardone ${ }^{1}$, V. Mellera ${ }^{1}$, D. Minelli ${ }^{1}$, M. Panella ${ }^{2}$, V. Piergotti ${ }^{2}$, P. Platania $^{1}$, G.Ramponi ${ }^{1}$, A. Simonetto ${ }^{1}$, B. Tilia ${ }^{2}$, E. Vitale ${ }^{2}$, O. Tudisco ${ }^{2}$

\footnotetext{
${ }^{1}$ Istituto di Fisica del Plasma CNR, Euratom Association, 20125 Milano, Italy

${ }^{2}$ Associazione EURATOM-ENEA, C.R. Frascati, Italy

${ }^{3}$ Politecnico di Milano, Dipartimento di Energia, Milano, Italy
}

\begin{abstract}
The Real-Time system for the control of the magnetohydrodynamics instabilities in FTU tokamak is presented. It is based on both a-priori information derived from statistical treatment of a database and Real-Time elaboration of live diagnostics data. The analysis codes are executed in different time threads based on multi-processors machines. The actuator is the $2 \times 0.4 \mathrm{MW} 140 \mathrm{GHz}$ ECRH system equipped with the new fast quasi-optical steerable launcher.
\end{abstract}

\section{Introduction}

A new Real-Time (RT) data acquisition and elaboration system is being implemented in FTU tokamak (major radius $\mathrm{R}=0.935 \mathrm{~m}$, minor radius, $\mathrm{a}=0.31 \mathrm{~m}$, max toroidal field $8 \mathrm{~T}$, max plasma current 1.6 MA, typical flat top pulse duration $1.5 \mathrm{~s}$ ) [1] to perform experiments of RT control of magnetohydrodinamic activity through a newly installed fast steerable two beams EC launcher [2]. The main goal of the system is the detection and control of $\mathrm{m} / \mathrm{n}=3 / 2$ and $2 / 1$ (neoclassical) tearing modes, (N)TM, and the control of the period of sawteeth activity in order to prevent the seeding of NTMs. However, the system architecture proposed and being implemented allows a range of applications, virtually extensible to all cases in which the steering of the ECH beams and their timely switching on/off linked with a suitable diagnostic signal or combination of signals could be desirable.

This paper is organized as follows. In section 2 the requirements for the design of the control system are exposed. The architecture of the RTC system and the functions performed by its components are illustrated in section 3. A brief description of the suite of numerical tools implemented, of their specific role in the system and of how they are used for MHD control is given in section 4 along with some experimental example. Conclusions follow in section 5.

\section{Guiding criteria, constraints and requirements}

In order to operate at high magnetic field FTU is equipped with a cryogenic toroidal field coil system [3]. The steel wall is also at cryogenic temperature $(77 \mathrm{~K})$. The outer cryostat imply severe constraints in the port geometry that reflects also on the auxiliary systems (including the ECRH antennae) and diagnostics characteristics, installation and maintenance [4, 5].

This is an Open Access article distributed under the terms of the Creative Commons Attribution License 2.0, which permits unrestricted use, distribution, and reproduction in any medium, provided the original work is properly cited. 
For such reason one of the driving criterion in the development of the control system has been the optimal use of the existing hardware including plasma diagnostics, adopting solutions able to compensate their limitations complementing the diagnostic data with other available information [6, 7]. The architecture of the system allows taking advantage of available a-priori information about the expected location of the instability. Such feature allows to optimize the response of the system and to supply a reliable status for the actuator even if the flow of diagnostic data is not regular. Detection, foreseeing and control of the action are performed on different hardware platforms working in separate time threads [8]. Finally, a modular implementation strategy has been chosen for both hardware and software. This allows to test step by step the new components.

The RT control system designed for FTU uses raw signals from the existing diagnostics, mainly electron temperature oscillations from a 12-channels ECE (Electron Cyclotron Emission) grating polychromator [9], 16 signals from the Mirnov coils system providing the fluctuation of the poloidal magnetic field [4] and 2 signals ECH microwave power monitors from directional couplers along the transmission line [10] (see scheme in figure.1). Other RT signals (plasma boundaries, plasma current $\mathrm{I}_{\mathrm{p}}$, toroidal magnetic field $\mathrm{Bt}$ and line integrated density) are obtained via the FTU plasma control system [11].

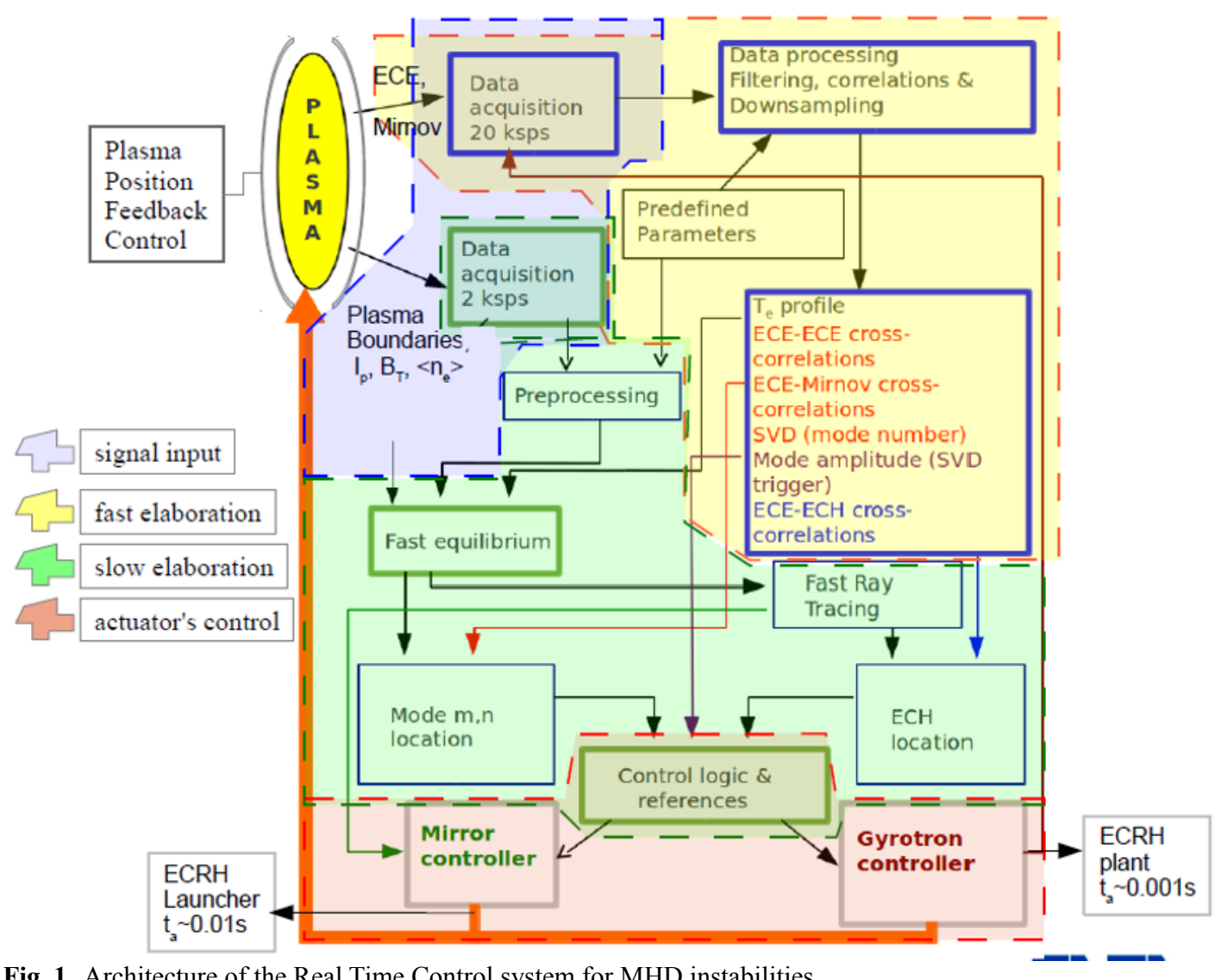

Fig. 1. Architecture of the Real Time Control system for MHD instabilities

ECE signals used alone or in combination with the magnetic signals are essentials in the presented architecture in order to detect the radial location of the MHD instabilities. At the magnetic field typical of ECRH operation $\left(B_{t}=5.3 T\right.$ at $\left.R=0.965 \mathrm{~m}\right)$ the spacing between channel is about $3 \mathrm{~cm}$, with radial resolution of $2 \mathrm{~cm}$. The typical size of FTU magnetic island is $2-3 \mathrm{~cm} \mathrm{[12],} \mathrm{rotating} \mathrm{at} \mathrm{1-}$ 
$10 \mathrm{kHz}$ frequency. Smallest islands can produce temperature oscillations below the detectable threshold of ECE.

The new launcher is fed by 2 out of the 4 x $0.4 \mathrm{MW} \times 0.5 \mathrm{~s} 140 \mathrm{GHz}$ gyrotron sources available. Switching from the previously installed launcher to the new one is performed through a pair of quasi optical switches manually actioned in shot-to-shot time intervals. The two beams launching points are symmetrical with respect to equatorial plane. Small size, low inertia steering mirrors allows angular speed $\Delta \theta / \Delta \mathrm{t}=100^{\circ} / \mathrm{s}$ (about $1^{\circ}$ or $1 \mathrm{~cm} / 10 \mathrm{~ms}$ at plasma axis) and a full poloidal (upper: $\alpha=:-15^{\circ},+50^{\circ}$; lower $\left.\alpha=:+15^{\circ},-50^{\circ}\right)$ or toroidal scan $\left(\beta=: \pm 40^{\circ}\right)$ in $400 \mathrm{~ms}$ with nearly independent movements along the two axis. The launcher is also equipped with a zooming system which allows variation of the beams size $\left(\mathrm{w}_{\min }=19.0 \mathrm{~mm}, \mathrm{w}_{\max }=26.0 \mathrm{~mm}\right.$ at the plasma axis $)$ [13].

\section{Architecture of the control system}

The software platform chosen to implement the control system is the MARTe (Multithreaded Application Real-Time executor) framework environment under RTAI Linux operating system. It runs on cPCI (and/or VME) industrial standard PCs hardware for both data acquisition and data elaboration using embedded motherboards equipped with quad core I7 processors.

The real time analysis tools and modules of the control logic are developed and tested in high level language such Matlab®, IDL®, F90 and then translated in C++ MARTe GAMs (Generic Application Module) maintaining their modularity. GAMs are easily managed \& combined to modify the control algorithm even in a shot-to-shot time scale [14].

The raw data from diagnostics are elaborated using the algorithms developed in order to detect the presence of the MHD activity, to track its radial position, to characterize its poloidal and/or toroidal mode number and to timely switch on/off the EC power aiming the beams towards the island. This set of signals undergoes a two-stage elaboration through the control chain. The flow of data is highlighted by the color code in figure 1. The blue area represents the data input. Fast diagnostic data (ECE, Mirnov, and ECH) are acquired with $20 \mathrm{KHz}$ sampling rate. Plasma current, toroidal field, line-averaged central density and magnetics for the last closed magnetic surface reconstruction are acquired at $2 \mathrm{KHz}$ sampling rate. After filtering and preprocessing, fast data feed the cross-correlation and the Single Value Decomposition algorithms, running in the fast time thread (yellow area). The output of the fast elaboration is transferred to the slow time thread (green area), together with data from the slow acquisition. The slow thread run the equilibrium reconstruction, the ray tracing and the main controller algorithms which provides the references signals for the mirror controller and for the gyrotrons power supply. Simple controller logics have been implemented and tested on off-line data [8].

\section{Location of the instabilities: a-priori-based and measurement-based}

The readjustment of the injection angle is the slower process in the control chain. Its overall time response can be considerably reduced introducing a tracking feature able to follow the probable location of the magnetic instability and reducing in this way the angular distance to be covered when the instability is actually detected. Such a function is performed by the fast equilibrium reconstruction FASTEQ based on a procedure of best fit of a large database (> 200) of FTU equilibria computed solving the Grad-Shafranov equation for a wide range of plasma parameters: $350 \mathrm{kA} \leq \mathrm{I}_{\mathrm{p}} \leq 850 \mathrm{kA}, 4 \mathrm{~T} \leq \mathrm{B}_{\mathrm{t}} \leq 7 \mathrm{~T}, 410^{19} \mathrm{~m}^{-3} \leq \mathrm{n}_{\mathrm{e}} \leq 1610^{19} \mathrm{~m}^{-3}, 1 \leq \mathrm{T}_{\mathrm{e}} \leq 8 \mathrm{keV}$ [15]. The map of poloidal flux surfaces is then obtained from RT signals of plasma boundaries (provided by magnetic measurements) and by an approximation of the plasma magnetic axis obtained by the thermal barycentre of the Te profile with suitable symmetrization provided by the interpolation of the polychromator channels: 


$$
R_{a x}=\int d R R T_{e} / \int d R T_{e}
$$

The dependence of the minor radius $\rho$ on $\psi_{\mathrm{n}}$ is best fitted as $\rho=\psi_{\mathrm{n}}{ }^{0.7}$.

The magnetic surfaces are parametrized as follows using the normalized poloidal flux $\rho$ and $0<\theta<2 \pi$ :

$$
\mathrm{R}=\mathrm{a} \rho \cos [\theta+\delta(\rho) \sin (\theta)]+\mathrm{RLCS}-\delta(\rho), \quad \mathrm{z}=\mathrm{a} \rho \sin (\theta) \mathrm{k}(\rho) .
$$

being $\mathrm{R}_{\mathrm{LCS}}$ the centre and a the radius in the equatorial plane of the last closed magnetic surface as estimated from the RT signals of the plasma boundary.
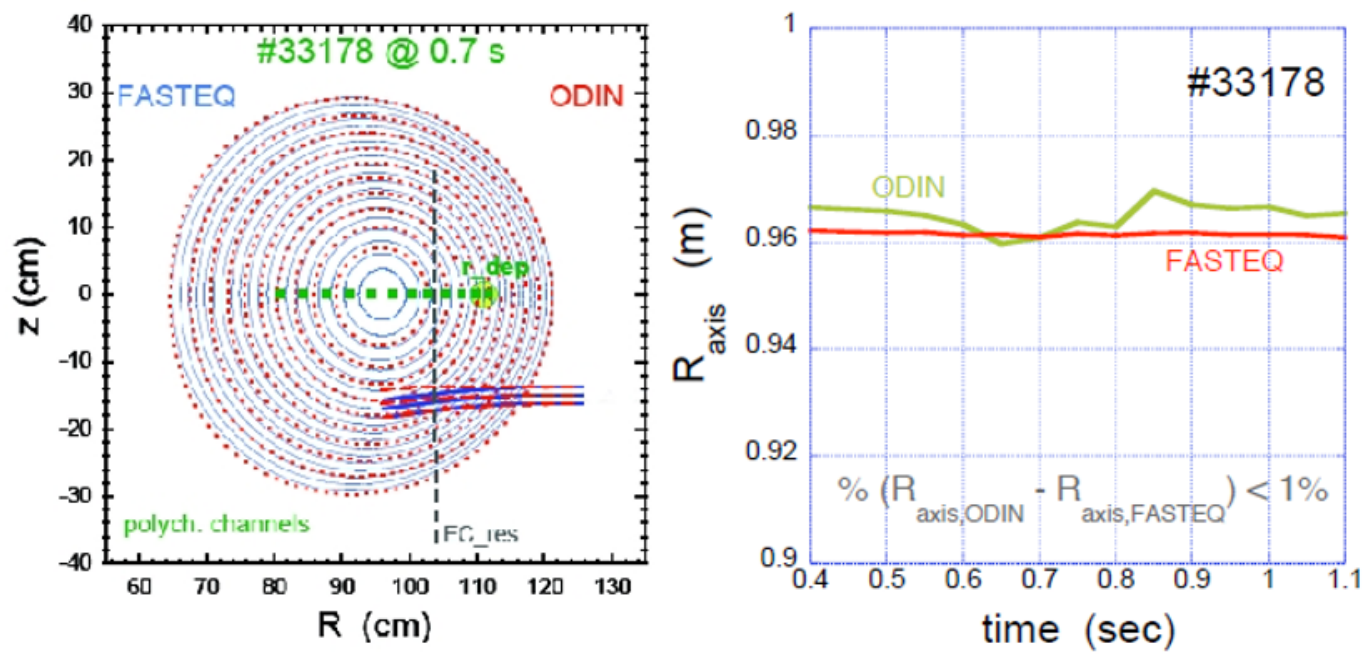

Fig. 2. Left: comparison of flux surfaces obtained by the FTU ODIN code (blue dashed lines) and the fast reconstruction FASTEQ (red lines) for FTU pulse 33178 at $\mathrm{t}=0.7 \mathrm{~s}$. Beam propagation from the lower launcher unit is shown as computed by FASTEQ (red) and by off line codes (blue). Right: evolution of the plasma axis as reconstructed from ODIN equilibrium code (green line) and from temperature barycentre (red line).

A reconstructed safety factor profile in cylindrical approximation $q(\rho) \approx 2 \pi \rho^{2} B_{t} / \mu_{0} I_{p}(\rho) R$ (where $R$ is the plasma major radius) can be derived as well using a fitted plasma currents profile $\mathrm{I}_{\mathrm{p}}$. The radius at which is expected the instability with mode numbers $\mathrm{m} / \mathrm{n}$ is then obtained equating $\mathrm{q}=\mathrm{m} / \mathrm{n}$.

A real time a-priori estimate of the EC power absorption location can be computed with the fast ray tracing RAYFAST [15] for a given pair of injection angles $\alpha$ (poloidal), $\beta$ (toroidal) defined such as $\tan (\alpha)=\mathrm{N}_{Z} / \mathrm{N}_{\mathrm{R}}$ and $\sin (\beta)=\mathrm{N}_{\phi}$ where $\mathbf{N}$ is the refractive index vector. The trajectory of the injected Gaussian beam is represented through three optical rays, the central one and 2 outer at the waist radial distance from the beam axis. The value of the absorption locations of these 3 rays gives the estimate of the a-priori EC deposition radius $\mathrm{R}_{\mathrm{dep}}$ and of the size of the step $\mathrm{dR} \mathrm{R}_{\mathrm{dep}} / \mathrm{d} \alpha$ to be considered for the subsequent actuator's poloidal readjustment. The difference between the values of $R_{\text {dep }}$ calculated with RAYFAST and with full beam tracing code such ECWGB [16] is $<5 \%$ (see figure 2). Typical execution times of the RT codes in their GAM version are reported in [8].

The actual presence of instabilities and their radial location is detected through the RT elaboration of diagnostics data samples. The cross-correlation of pairs of signals is evaluated at the sampling rate $(20 \mathrm{kHz})$ [17]: ECE radially adjacent polycromator's channels; one ECE channel and one selected Mirnov coil; one ECE and the ECRH power signal. The first two pairs provide 11 (or 12) time-dependent cross-correlation coefficients as the example shown in figure 3 for pulse 34742. The change of sign in the cross-correlation coefficients (corresponding to a jump of phase of about $\pi$ ) of radially adjacent channels indicates the radial location of the island. The third one (applied only during ECRH power modulation) indicates the radial location of the ECRH absorption. The SVD 


\section{EC-17 Workshop}

(Singlular Value Decomposition) is applied on the signals of toroidal and poloidal sets of Mirnov coils and is based on the phase difference among the different coils [18].

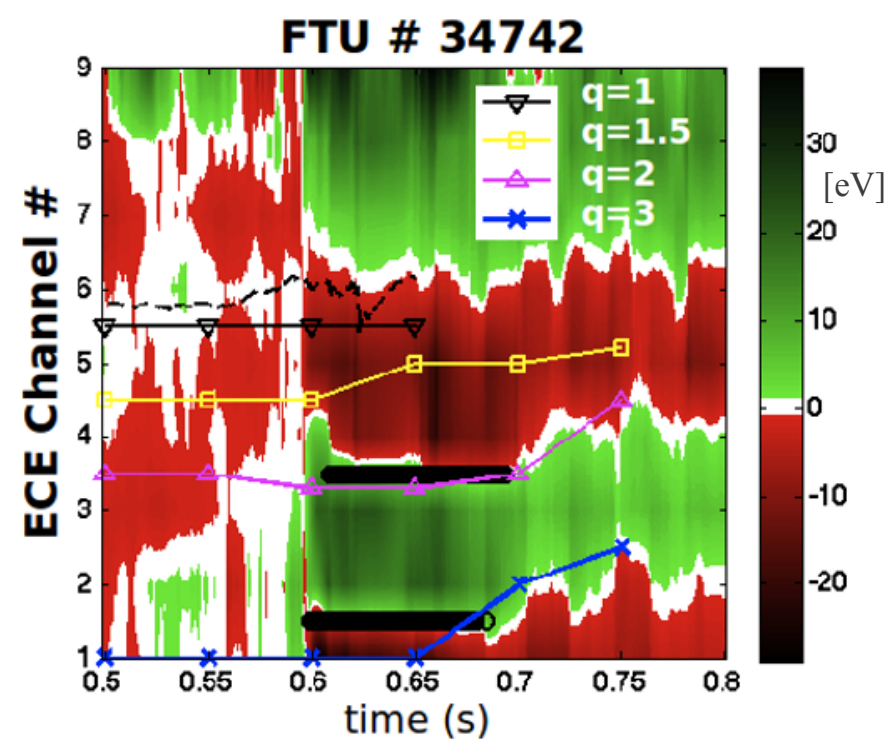

Fig. 3. Map of the amplitude (in eV) of the cross-correlation coefficients between Mirnov coil (ch. 13) and ECE channels vs time for FTU shot 34742. Black circles indicate mode detection via change of sign of cross-correlation with amplitude beyond a given threshold $(19.8 \mathrm{eV})$. Colour lines mark RT-computed rational q surfaces. The black dashed line indicated the thermal barycentre of plasma ( magnetic axis).
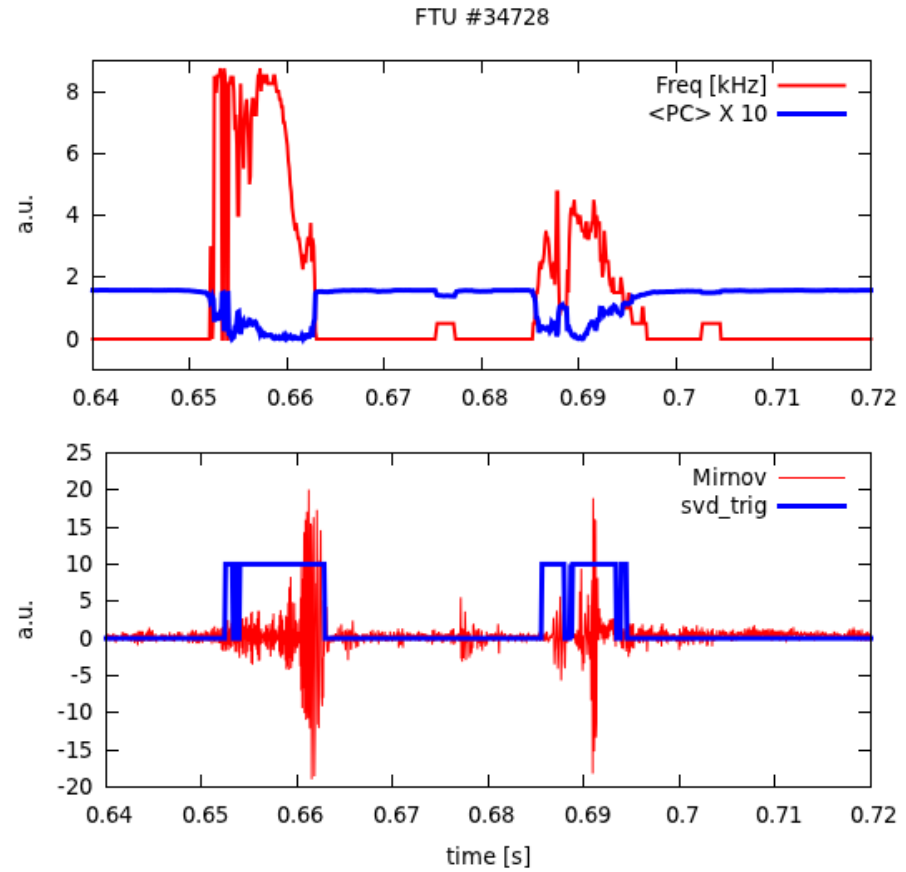

Fig. 4. Top: frequency (red line) and average of PC. Bottom: amplitude of one the Mirnov coil (red) and behaviour of a trigger signal generated by the SVD algorithm when $<\mathrm{PC}>$ falls below threshold. 
Its implementation for RT control has been originally motivated for $\mathrm{m}, \mathrm{n}$ mode radial localization in combination with the equilibrium reconstruction [19]. Although this application is not straightforward, SVD is proving rather effective in the early detection of mhd activity as figure 4 shows. Both the behaviour of the dominant Principal Component (PC, representing the mode in which the time series of the Mirnov signals oscillate longer in the given data packet) and its frequency are sensitive in respect of the amplitude of the instability as detected by one single coil. In fact when the instability builds-up quickly the average $\langle\mathrm{PC}\rangle$ vanishes and the frequency reaches the typical range of the tearing instabilities in FTU.

\section{Conclusions}

The architecture of the Real-Time system for NTM control on FTU has been finalized and most of the key components have been developed. The algorithms of elaboration of the diagnostics data, the a-priori algorithms for pre-alignment of the beams and simple controller logics have been implemented and tested on off-line data. In the first operations performed on a plasma several elements of the control chain have been tested.

\section{Acknowledgments}

This work was supported by EURATOM and carried out within the framework of the European Fusion Development Agreement. The views and opinions expressed herein do not necessarily reflect those of the European Commission.

\section{References}

1. C. Gormezano et al. Fus. Sci. Techn. 45, 3, 297-302 and 303-322 (2004)

2. A. Bruschi et al., Fus. Sci. Tech. 55, 1, 94-107 (2009)

3. A. Pizzuto et al., Fus. Sci. Techn. 45, 3, 422-436 (2004)

4. O. Tudisco et al. Fus. Sci. Techn. 45, 3, 402-421 (2004)

5. M. Aquilini et al. Fus. Sci. Techn. 45, 3, 459-482 (2004)

6. G. D'Antona, S.Cirant, M.Davoudi, IEEE Trans. on Nuclear Science, 58, 4, 1503-1510 (2011)

7. S.Cirant et al., Fus. Scie. Tech., (53), 1 174-183 (2008)

8. C. Galperti et al., this conference.

9. O. Tudisco et al., Rev. Sci. Instrum., 67, 9, 3108-3116 (1996)

10. A. Simonetto et al., Fus.Eng.Des., 53, 1, 301-308, (2001)

11. Y. Sadeghi et al., IEEE Trans. on Plasma Science, 38, 3, 352-358 (2010)

12. P. Buratti et al., Fus. Sci. Techn. 45, 3, 350-369 (2004)

13. A. Moro et al., this conference.

14. L. Boncagni et al., Fus. Eng. Des., 86, 6-8, 1061-1066 (2011)

15. S. Nowak et al., Proc. $38^{\text {th }}$ EPS Conf. Contr. Fusion and Plasma Phys., Strasbourg, France, P4085 (2011)

16. S. Nowak and A. Orefice, Phys. Plasmas 11242 (1994)

17. E. Alessi, et al. this conference. See also: E. Alessi et al., Proc, of 2nd Intnl. Conf. on Frontiers in Diagnostic Technologies, (2011), submitted for publication, Elsevier

18. C. Nardone, Plasma Phys. Contr. Fus. 34, 1447-1465 (1992)

19. C. Marchetto et al, Proc. $37^{\text {th }}$ EPS Conf. Contr. Fusion and Plasma Phys., Dublin, ECA 34A, P5117 (2010) 